\title{
Reimbursement for Teledermatology During the COVID-19 Public Health Emergency: Change Has Come, But Will It Stay?
}

George Han, MD, PhD

\rceil he world of telemedicine-especially teledermatology — had been a sleepy underutilized afterthought for most physicians until we were faced with a global pandemic the likes of which none of us had seen in our lifetimes. And just like that, teledermatology went from an afterthought to part of the "new normal." Although those of us already practicing telemedicine knew of potential pitfalls and concerns, this great social experiment of throwing everyone into unexplored territory led to a great deal of frustration with technology and workflows that were not optimized for dermatology visits. The process is still changing, and the technical aspects of conducting teledermatology visits will no doubt improve, but what about the bigger question of reimbursement? Without adequate payments and financial models, the long-term future of telemedicine is uncertain, so an understanding of the current and likely future landscape of telemedicine reimbursement is critical.

\section{Waivers During the Public Health Emergency}

The declaration of a public health emergency (PHE) allowed for significant flexibility by the Centers for Medicare \& Medicaid Services (CMS) during the coronavirus disease 2019 (COVID-19) pandemic. Importantly, the CMS was permitted to act quickly to allow telehealth to flourish during the worst of the pandemic and throughout the declared PHE, which has been extended several times already. Currently, the PHE is set to expire on April 20, 2021, but may be extended again if the pandemic is ongoing. The most important of these waivers was probably the removal of both the originating site and geographic requirements for telehealth services. ${ }^{1}$ Prior to the COVID-19 PHE, a patient would have to travel to a doctor's office, hospital, or skilled nursing facility to receive telehealth care (originating site requirement), and even then this was only allowed in defined rural areas of the country (geographic requirement). Both of these requirements were waived, allowing for any patient to receive telehealth services within their own homes. Concurrently, the requirement that patients must have an established relationship with the provider (ie, telehealth could not be used to provide care to new patients) also was waived. ${ }^{1}$

In the spirit of expanding access to care and providing reasonable reimbursement for medical services, other changes were made for which the CMS should be commended. In acknowledging that many Medicare/ Medicaid beneficiaries may not have access to devices that permit real-time, 2-way audio/video communication, which previously were necessary to qualify for a telehealth encounter, the CMS decided to cover telephone visits and provide reimbursement at the level of an established visit. ${ }^{1}$ They also changed the billing structure to remove the place of service (POS) designation for telehealth (POS 02) and replace it with the normal physician's office POS designation (usually POS 11), bringing back a telehealth modifier (modifier -95) in the process. The benefit of this change is solely to increase reimbursement for these services, as telehealth POS services generally are covered at lower facility rates, whereas POS 11 codes are reimbursed at the full level of a nonfacility physician's office rate.

Finally, other waivers such as the Office of Civil Rights' decision to waive HIPAA (Health Insurance Portability 
and Accountability Act) violations for telehealth platforms during the PHE allowed offices to take on telemedicine quickly without having to implement a new infrastructure. ${ }^{2}$ Numerous codes were added to the list of covered services for telehealth, but these generally are not relevant for dermatologists. The CMS also allowed physicians' offices to waive the patient responsibility/ co-pay during the COVID-19 PHE, which previously was not allowed due to concerns about the anti-kickback statute. ${ }^{1}$ These co-pay waivers were intended to remove another barrier to care for patients who were hesitant to participate in virtual visits. For the most part, the waiver of state licensing requirements is a bit less useful. As part of the CMS waiver, providers technically are allowed to see out-of-state Medicare/Medicaid beneficiaries, but state licensing laws are still in effect; thus, in the absence of a blanket state-level waiver (which some states enacted, modeled after the Uniform EmergencyVolunteer Health Practitioner Act of $2006^{3}$ ), providers still cannot see most out-of-state patients from a legal and malpractice coverage standpoint.

An important flexibility during the COVID-19 PHE is one that often is underrecognized. The CMS has been clear about the ability to provide direct supervision for advanced practice providers (APPs) and residents via telehealth during the PHE, which allows for incident-to billing for APPs at remote sites given that the supervising physician is immediately available via an interactive, 2-way, live audio/video telecommunications method. It also allows for direct supervision of APPs and residents using such technology. For dermatology, which does not have a primary care waiver, an attending must still directly supervise each patient and see the patient via a live audio/video modality but does not have to be onsite to do so. This is a very interesting concept that, if extended, could truly impact practice management for the long-term.

\section{Response From Commercial Insurance Carriers}

Tracking along with the CMS waivers and flexibilities during the PHE, most commercial carriers quickly adopted similar policies to cover telehealth services. It should be noted that for most commercial insurance carriers, the coverage was already broader than Medicare/ Medicaid coverage for telehealth prior to the PHE, so in many ways it is an extension of that concept and acceptance of telemedicine as a whole. What is sometimes confusing, though, is that various policies and requirements around billing exist; for example, while most carriers emulated the POS requirements that the CMS adopted, some carriers still stuck with the telemedicine POS but paid full in-office visit rates for those codes. Some carriers adopted higher reimbursement rates for telephone visits, similar to the CMS, while others instructed providers to just bill for the established office visit codes and allowed for telephone-only visits to qualify for these billing codes. Some carriers also waived co-pays for telehealth visits for their members (whether related to COVID-19 or not). It is beyond the scope of this article to delve into the specifics, which may vary not only by carrier but by region and plan. However, it is important to stay on top of one's insurance carriers to find out what their latest directives are for billing for telehealth.

\section{Postpandemic Teledermatology}

What about the future of teledermatology? Although many dermatologists have adopted telehealth services out of necessity during the COVID-19 PHE, the jury is still out on the long-term forecast for telemedicine in dermatology. Concerns about liability/malpractice and technology issues abound, and for many, the headaches of teledermatology — such as trying to focus on a blurry photograph of a nevus that the patient is concerned about-make it unappealing. Some of these issues will be addressed by better technology, but the reimbursement structure must continue for teledermatology to remain in widespread use.

Currently, the biggest question facing telehealth is whether the waivers for originating site and geographic requirements will be able to continue. The CMS itself does not have the statutory authority to make these changes permanent and was only allowed to act due to a waiver under section 1135 of the Social Security Act during a PHE. It would take an act of Congress to change the law to allow for this specific expansion of telehealth services. A number of federal bills, including S 2741 (Creating Opportunities Now for Necessary and Effective Care Technologies [CONNECT] for Health Act of 2019) and S 4796 (Fair Care Act of 2020) from the Senate, contain such provisions, but none have been passed at the time of writing. There does seem to be broad support of the concept of expanding telemedicine access, such as noted by New York State Governor Andrew Cuomo in his 2021 State of the State address, ${ }^{4}$ but it remains to be seen when action will come.

Some regulations, such as the HIPAA waiver and the ability to waive co-pays, are not slated to continue after the pandemic. The ability to supervise residents via telehealth (real-time audio/video) has been made permanent, but only in rural areas. Direct supervision of APPs via telehealth will continue through the end of the calendar year of the PHE or the end of 2021, whichever comes later, but it remains to be seen whether remote supervision will continue. The CMS has stated in its comments that it is looking at this issue closely and may establish certain guardrails to ensure quality of care is maintained. ${ }^{1}$ Telephone/audio-only visits also may come under further scrutiny, but research has supported the concept that patients who are more likely to gain access through audio-only modalities are older, Medicare/Medicaid (vs commercial), and Black (vs White) patients, ${ }^{5}$ so it would indeed introduce an unfair barrier to access if such coverage was rolled back.

CONTINUED ON PAGE 70 


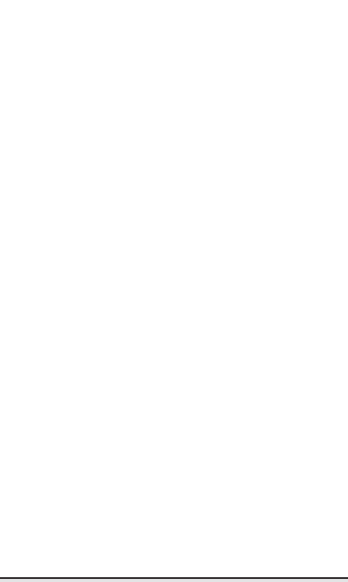

EDITORIAL

CONTINUED FROM PAGE 62

\section{Final Thoughts}

Overall, we have made much progress in teledermatology. Once utilized by a small fraction of dermatologists, the vast majority of us turned to teledermatology to sustain our practices during the COVID-19 pandemic. Moving forward, there are 2 critical factors to consider: continued technological innovation and permanent coverage for telehealth reimbursement at in-office visit levels. With these challenges resolved, we can move forward and consider novel models that may be able to deliver dermatologic care to a broader patient population, thereby solving the critical issue of access to care for so many patients in need in our country.

\section{REFERENCES}

1. Medicare Program; CY 2021 Payment Policies Under the Physician Fee Schedule and Other Changes to Part B Payment Policies; Medicare Shared Savings Program Requirements; Medicaid Promoting Interoperability Program Requirements for Eligible Professionals; Quality Payment Program; Coverage of Opioid Use Disorder Services Furnished by Opioid Treatment Programs; Medicare Enrollment of Opioid Treatment Programs; Electronic Prescribing for Controlled Substances for a Covered Part D Drug; Payment for Office/Outpatient Evaluation and Management Services; Hospital IQR Program; Establish New Code Categories; Medicare Diabetes Prevention Program (MDPP) Expanded Model Emergency Policy; Coding and Payment for Virtual Check-in Services Interim Final Rule Policy; Coding and Payment for Personal Protective Equipment (PPE) Interim Final Rule Policy; Regulatory Revisions in Response to the Public Health Emergency (PHE) for COVID-19; and Finalization of Certain Provisions from the March 31st, May 8th and September 2nd Interim Final Rules in Response to the PHE for COVID-19. Fed Registr. 2020;85:84472-85377. To be codified at 42 CFR §400,410,414, 415, 423, 424, and 425 . https://www.federalregister.gov/documents/2020/12/28/2020-26815 /medicare-program-cy-2021-payment-policies-under-the -physician-fee-schedule-and-other-changes-to-part

2. Office for Civil Rights. Notification of enforcement discretion for telehealth remote communications during the COVID-19 nationwide public health emergency. US Department of Health and Human Services website. Reviewed January 20, 2021. Accessed January 25, 2021. https://www.hhs.gov/hipaa/for-professionals/special-topics /emergency-preparedness/notification-enforcement-discretion -telehealth/index.html

3. Hoffman DA. Increasing access to care: telehealth during COVID-19 [published online June 16, 2020]. J Law Biosci. doi:10.1093/jlb/lsaa043

4. Governor Cuomo announces proposal to expand access to telehealth for all as part of 2021 State of the State. New York State website. Published January 10, 2021. Accessed January 25, 021. https://www.governor.ny.gov/news/governor-cuomo-announces - proposal-expand-access-telehealth-all-part-2021-state -state\#: : text=and \% 20Rural \% 20Communities-, Governor $\% 20$ Andrew\%20M.,2021\%20State\%20of\%20the\%20State.\&text=New\%20 Yorkers \% 20have \% 20adapted \% 20throughout, in to \% 20 our \% 20 existing\%20healthcare\%20system

5. Gilson SF, Umscheid CA, Laiteerapong N, et al. Growth of ambulatory virtual visit and differential use by patient sociodemographics at one urban academic medical center during the COVID-19 pandemic: retrospective analysis. JMIR Med Inform. 2020;8:E24544. 